\title{
Building a Network of Biological Resource Centers for Research Purposes in the Caribbean: Excellent Potential for Research into Public Health Diseases
}

\author{
S. Gaete ${ }^{1, *}$, J. Deloumeaux $x^{1,2,3}$ \\ ${ }^{1}$ Biological Resource Center, University Hospital of Guadeloupe, French West Indies \\ ${ }^{2}$ Delegation for Clinical research and Innovation, University Hospital of Guadeloupe, French West Indies \\ ${ }^{3}$ Cancer Registry of Guadeloupe, University Hospital of Guadeloupe, French West Indies
}

Copyright $\bigcirc 2017$ by authors, all rights reserved. Authors agree that this article remains permanently open access under the terms of the Creative Commons Attribution License 4.0 International License

\begin{abstract}
Biological Resource Centers (BRCs) and their associated molecular biology platforms are the keys to the development of clinical research, by enabling the utilization and sharing of dedicated collections of human biological samples. Whether in the aim of identifying biomarkers for existing diseases, or improving knowledge of the pathophysiology of emerging diseases, these collections have the ultimate goal of improving patient care. The BRC of the University Hospital of Guadeloupe calls for the implementation of a Caribbean network between BRCs and Biobank-type structures across the Caribbean, in the following areas: cancer, infectious and emerging diseases, hematological, and cardio-metabolic diseases. This Caribbean Network of Biological Resource Centers (CNBRC) aims to become a key player in proposing specific ad hoc research topics, bringing together biological materials from Caribbean populations with similar health problems. A network of this type would facilitate academic and/or industrial partnerships, promote exchange of know-how through dedicated platforms, and link research teams through communication tools. Furthermore, collaboration between plant and animal BRCs could enable preclinical studies on Caribbean flora and fauna, and their impact on human health. Sharing expertise and processes, and ensuring a reliable delivery system for quality biological samples, would be at the core of the network's functioning. Therefore, setting up a CNBRC represents a unique opportunity to expand the scale and quality of clinical research in the Caribbean in two ways, namely:-firstly, by bringing together data from populations who share geographic, environmental and genetic similarities, but which may not have sufficient sample sizes to yield the required statistical power when studied separately; -secondly, by giving local scientists the tools to develop the expertise within their own communities.
\end{abstract}

Keywords Biological Resource Centers, Network, Caribbean, Human Samples, Research

\section{Introduction}

Modern medical Research relays mostly on epidemiologic studies or clinical trials, framed by Good Clinical Practices. Over the last decades, the biotechnological advances, with genome and epigenetic studies (RNAs, Proteins, metabolites...) have led to an increasing use of existing biological data collections mostly set up by researchers for their own purpose. To increase the power of their observations, researchers have created networks to share biological collections around pathologies of interest [12]. More and more structured and regulated, these collections joined within biological resources centers have reached however some limits. The lack of representativeness of some of these biological data when studying biomarkers linked to population characteristics questioned the need for dedicated collections.

In the Caribbean, which populations are mainly of African descent, non-communicable diseases (obesity, cardiovascular disease, Hypertension, diabetes and cancer) and infectious diseases have some specific epidemiological characteristics [1;17]. Still these populations are seldom represented in most international studies.

In Guadeloupe particularly, diseases identified as public health priorities for collecting biological resources are quite like to those observed in other Caribbean countries as shown in Figure 1. 


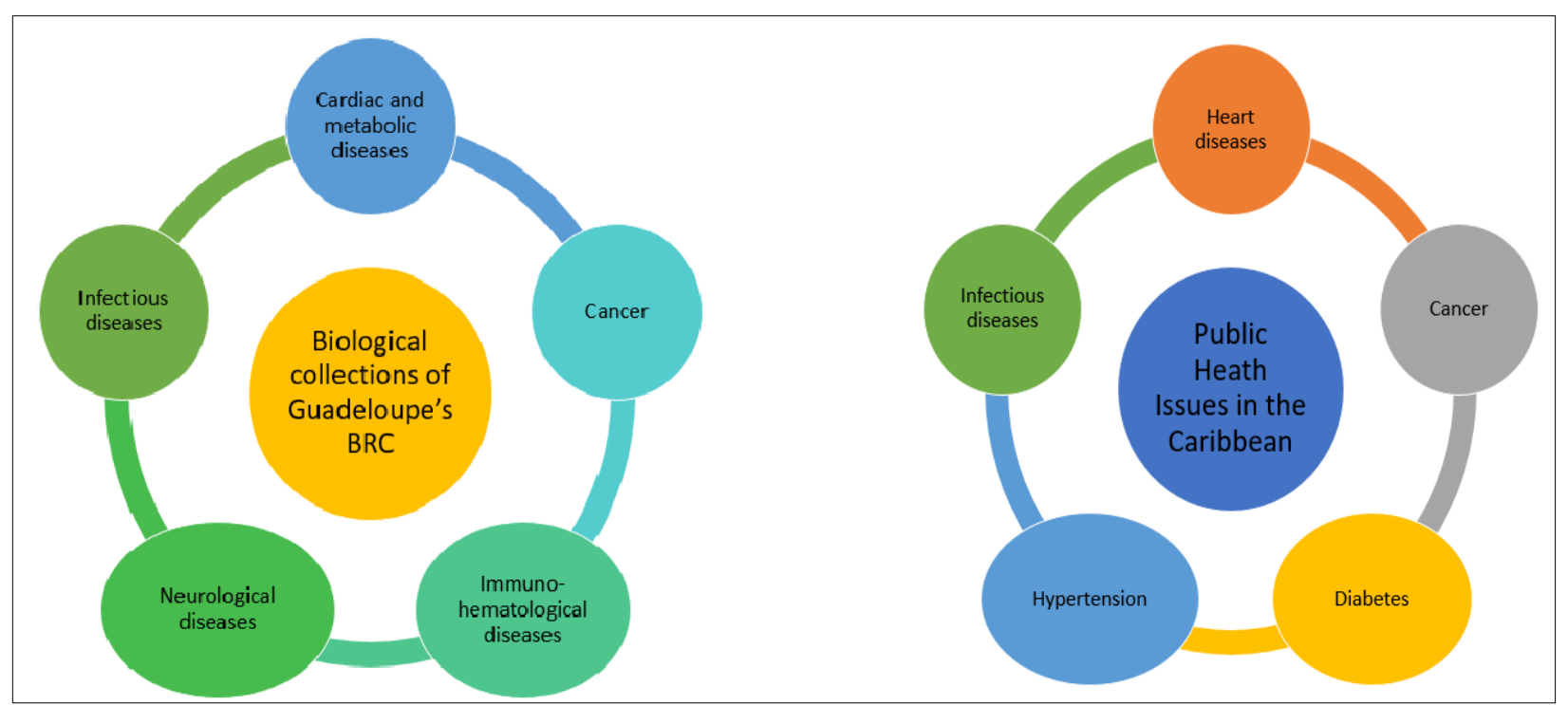

Figure 1. Relationship between public health issues in the Caribbean (CARPHA source) and collections reported to the Biological Resource Center of Guadeloupe

Yet few pooled studies are conducted within these populations. As French territories, most studies conducted in Guadeloupe and/or Martinique are usually associated or compared to mainland France data. In recent years however, openings toward African countries were nevertheless observed; mainly for hematologic disease such as sickle cell anemia [21] and prostate cancer research [20] both highly prevalent in our population as well as in Africa.

Though living standards is different between most English-speaking countries and French-speaking territories of the Caribbean (that includes French Guyana, Guadeloupe and Martinique) the geographical and genetics patterns are very similar to their neighborhoods' in the Caribbean. Finally, the small sizes of most Caribbean countries have to be taken into account when considering larger scale studies, especially in genetics and epigenetics.

Racial differences in risk factors and prognosis have been reported for many diseases [2]. The documented racial differences in heart failure $[13 ; 15 ; 16]$, and the aggravating factors in African, and afro-descent populations of the Caribbean, highlight the need for joined biomolecular mechanisms analyses is obvious.

The genetic particularities of populations of Afro-descent have also been demonstrated for immune system, breast cancer, etc...) in research projects mainly in the USA $[5 ; 6$; 7]. A few of these studies rely on substantial biological samples from Caribbean individuals to clarify the genetic susceptibilities in this population, and the impact of environmental factors.

In addition, the Caribbean is also an endemic area for the emergence of viral pathogens (carried by mosquitos, or by sexual activity ...). The recent outbreak of Chikungunya and Zika virus in the Caribbean and South America highlights the need for reactivity, sharing of information and data pooling bringing the World Health Organization to define a strategic priority, named Health information systems and research
[8].

This article presents the necessity of a network of human biological sample collector centers to improve medical research in the Caribbean.

\section{The challenge of pooling researches in the Caribbean}

Although the need of more collaboration is identified by many organizations, the links between professionals and structures is not completely formalized and relay mainly on individuals. Studies based on Caribbean populations clinical data as well as biological samples are requested, but researchers have to overcome some significant challenges. First the geographical one: the Caribbean health organizations includes the following countries: Antigua and Barbuda, Anguilla, The Bahamas, Barbados, Belize, Bermuda, the British Virgin Islands, the Cayman Islands, Dominica, Grenada, Guyana, Jamaica, Montserrat, Saint Lucia, St. Vincent and the Grenadines, St. Kitts and Nevis, Suriname, Trinidad and Tobago, and the Turks and Caicos Islands. The greater Caribbean also covers the French West Indies, Cuba, Haiti and St. Domingue.

These countries have different population size, languages, laws and socioeconomic level. Although, the Pan American Health Organization (PAHO) produces epidemiological indicators (incidence, mortality, morbidity and risk factors) for these Caribbean countries through the Caribbean Public Health Agency (CARPHA), an accurate knowledge of prevalent and/or emerging diseases in these territories are needed [10].

This biobank network project will have an important role to play in this program, with biological samples that could support research projects and generate data on a range of health topics relevant to Afro-Caribbean people (Figure 2). 


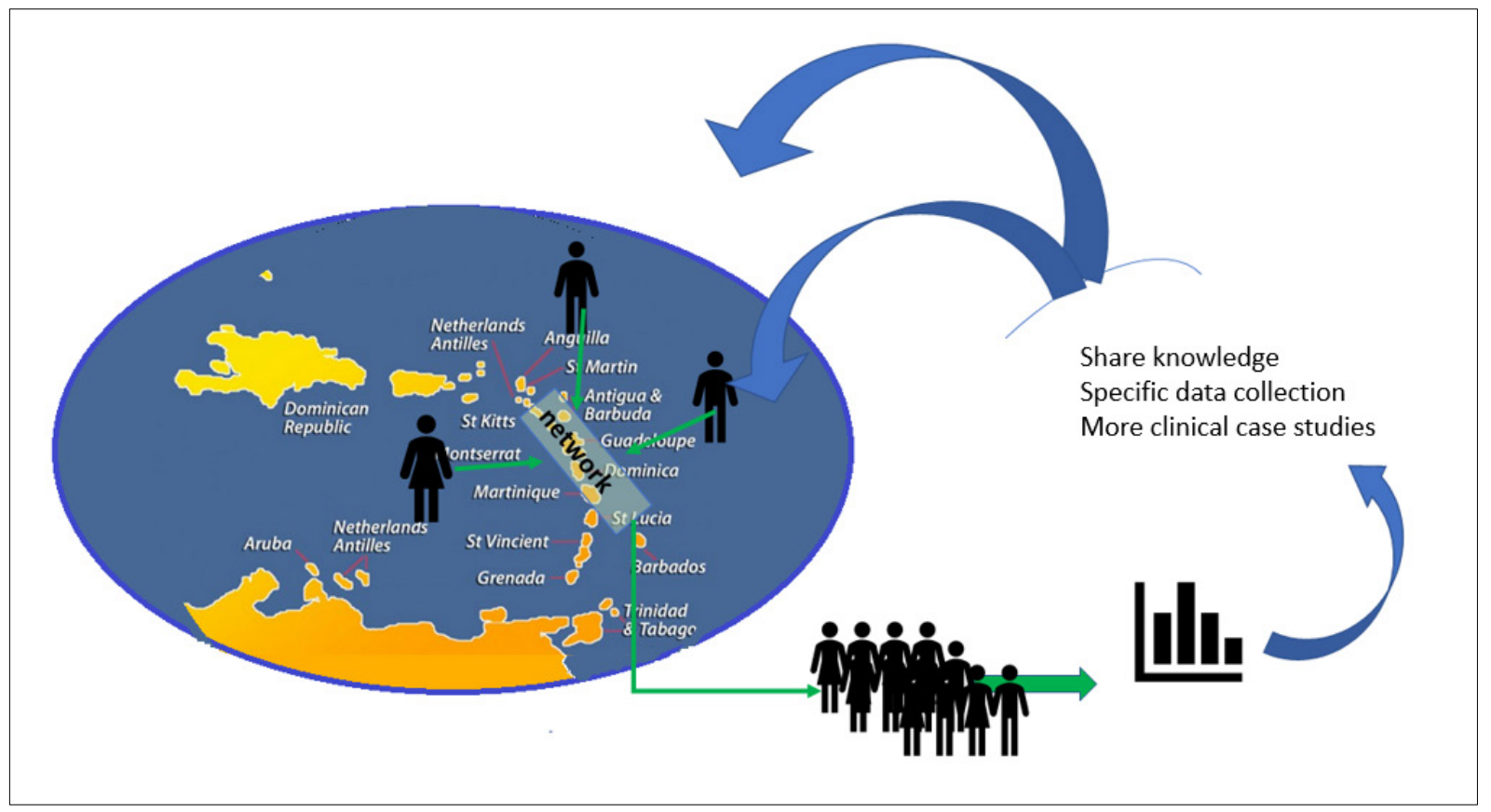

Figure 2. Virtuous circle of Research Improvement in a Caribbean Network of BRC

The advent of this network will make possible to identify both risk and prognostic factors in these populations taking into account their geographic and genetic susceptibilities. Harmonized procedures are necessary, to allow easy exchanges between the different organizations (i.e.: Caribbean Community CARICOM, Healthy Caribbean Coalition HCC, PAHO, and French delegation of Clinical Research) lead by their country of attachment.

The BRC of Guadeloupe is already involved in local, national and international research projects. There is nevertheless a compelling need to develop greater collaboration on the subject of the public health impact of certain diseases in the Caribbean (i.e. cancer, cardiac diseases, infectious and environmental diseases, immune, hematologic and neurologic diseases), figure 3.

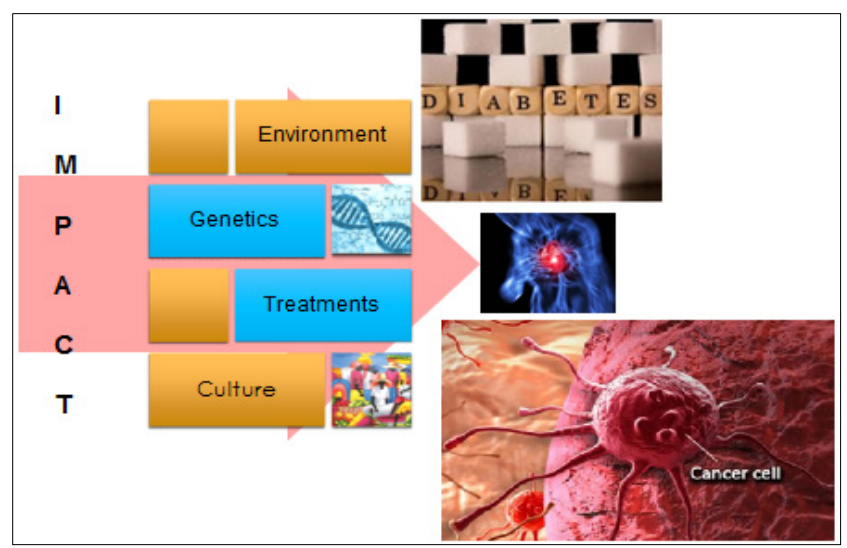

Figure 3. Different factors influencing acute diseases in the Caribbean

Setting up biological collections is challenging, particularly in the absence of financial resources. The support of existing biological resources structures as BRC of Guadeloupe could be useful for managing and hosting collections as a first step in the network.

To bring together researchers around Caribbean topics, it will be necessary:

- To develop a specific catalog of biological resources from populations with genetic diversity.

- To improve our understanding of the molecular and cellular mechanisms of disease and their impact on incidence, severity and mortality.

- To develop industrial collaborative projects, for example screening tests or sensitivity / specificity diagnostic tests.

- To develop specialized platforms and share know-how in molecular and cellular biology (testing kits, high throughput screening of viral genotypes or mutation testing in non-communicable diseases ...)

- To develop collaboration with other types of BRC (plants, animals) in translational model studies on the impact of flora and fauna on human health.

\section{Set up and Management of a Biobank Network in the Caribbean}

Worldwide, biobank networks have multiplied in the last decade. In Europe, existing networks include the European Middle Eastern and African Society for Biopreservation and Biobanking (ESBB) [3] and the Biobanking and Biomolecular Resources Research Infrastructure (BBMRI) [4].

In France, the main networks are represented by "Biobanques" led by the French National Institute for 
Medical Research (INSERM) and the "Club 3-CR" [9]., which group more than 95 biobanks. These structures are governed by the Ministry for Higher Education and Research, and are subject to a charter and national standards (NFS 96900) based on ISO 9001 and ISO 17025. BRCs work in respect to bioethics guidelines describing procedures for the management and preparation of biological samples as well as the transfer of biological resources for research outcomes [14; $18]$.

In recent years, BRCs have combined bio banking and laboratory research activities that placed them at the center of many advanced projects, especially in epidemiology. The BRC of Guadeloupe has set up Molecular Biology technics (DNA, RNA extraction, immunoassay, specific hybridizations, Polymerase Chain Reaction (PCR), Real Time PCR...) based on state of the art technics [18], available for local researchers but also for Caribbean partners.

A Caribbean network could therefore benefit from the expertise of these existing networks and be linked to some of these national or internal networks for specific projects and requests. The originality and relevance of this network will rely on the wide genetic diversity of its populations. Indeed, most African-Caribbean's have ancestries from Benin, Nigeria, Ghana and Senegal because of the transatlantic slave trade and may share common polymorphism [19].

From a legal standpoint, while each biobank has to obtain authorization from their local ethics committee, it will be necessary to harmonize the process with a dedicated ethics committee. Its role will be to ensure that all participating organizations respect the legislation in regards to patient information and consent.

The main point of the network being to provide researchers with high-quality biological material and related data, we will also need high quality transmission of both the material and related data. A secure, joint exchange format should be used within the network to facilitate the exchanges between the Caribbean islands. Furthermore, the shipment of resources should be performed in accordance with IATA guidelines and subcontracted to certified express shipping services, given the geographic spread of the Caribbean.

\section{Conclusions}

The idea of a Caribbean biobank network project was first proposed at the 20th conference of the Caribbean Academy of Sciences in November 2016 by the BRC of Guadeloupe [11]. Many health organizations are being approached both locally (Regional Health Agency in Guadeloupe), in the Caribbean (Caribbean Public Health Agency, Pan American Health Organization) and internationally (National Institute on Minority Health and Health Disparities, NIMHD). This approach aims to open new ways of research with associated biological collections, for the benefit of patients internationally.
Our challenge to build a Caribbean network relies on a relevant scientific foundation, as well as a compelling need in the context of global data pooling (Next-Generation Sequencing and genome-wide association studies).

\section{Acknowledgements}

We are very grateful to the University Hospital of Guadeloupe.

We are very grateful to Caribbean Academy of Science. We are very grateful to the team at the Biological Resource Center of the University Hospital of Guadeloupe.

We are very grateful to $3 \mathrm{C}-\mathrm{R}$.

\section{REFERENCES}

[1] Delgado-Cruzata L et al. Dietary modifications, weight loss, and changes in metabolic markers affect global DNA methylation in Hispanic, African American, and Afro-Caribbean breast cancer survivors, 2015, DOI:10.3945/jn.114.202853

[2] Bennett NR, Francis DK, Ferguson TS, Hennis AJ, Wilks RJ, Harris EN, MacLeish MM, Sullivan LW; U.S. Caribbean Alliance for Health Disparities Research Group (USCAHDR),Disparities in diabetes mellitus among Caribbean populations: a scoping review, 2015, DOI:10.1186/s12939-015-0149-z

[3] The Research Biobank of the Year Competition of the European, Middle Eastern and African Society for Biopreservation and Biobanking (ESBB): aims and achievements , 2014 , DOI:10.1089/bio.2014.0004

[4] BBMRI-ERIC: the novel gateway to biobanks. From humans to humans, 2016, DOI:10.1007/s00103-015-2301-8

[5] M. Campbell et al. AFRICAN GENETIC DIVERSITY: Implications for Human Demographic History, Modern Human Origins, and Complex Disease Mapping,2008, doi: 10.1146/annurev.genom.9.081307.164258

[6] Nédélec Y et al. Genetic Ancestry and Natural Selection Drive Population Differences in Immune Responses to Pathogens, 2016, DOI:10.1016/j.cell.2016.09.025

[7] Bernadette Theodore-Gandi. Protecting and Improving the Health of the Caribbean People, DOI:10.2105/AJPH.2007.131086

[8] PAN WHO Subregional Cooperation Strategy 2016-2019 Report. Available online from http://www.paho.org

[9] J. Di donato, S.Gaete ${ }^{1}$, Openness to the world of BRC, The keys to understanding (Original title: Ouverture sur le monde des CRB les clés de la comprehension), 3C-R Edition, France, 2015. ISBN 2953361324, 9782953361322

[10] PAHO/WHO, CARICOM, Report of the Caribbean Commission on Health and Development

[11] Yves Mazabraud, Thomas Forissier, Alain Pietrus, Olivier Gros, Claire Anjou. CARIBBEAN ACADEMY OF 
SCIENCES 20th GENERAL ASSEMBLY AND BIENNAL MEETING: Planning and Abstracts book. Nov 2016, Deshaies, Guadeloupe, 56, 2016. hal-01413318.

[12] Durski et al. Development, Use, and Impact of a Global Laboratory Database During the 2014 Ebola Outbreak in West Africa, J Infect Dis., 2017

[13] Bibbins-Domingo K et al. ,Racial differences in incident heart failure among young adults, 2009 New England J Med.1179-1190

[14] J Med Ethics, Councils for International Organizations of Medical Science, 2001

[15] Monika M.Safford et al. Association of Race and Sex With Risk of Incident Acute Coronary Heart Disease Events , JAMA 2012 1768-1774

[16] Okin P.M et al. Racial differences in sudden cardiac death among hypertensive patients during antihypertensive therapy: the LIFE study; Heart Rhythms; 2012; 531-537.

[17] Ian R. Hambleton et al. Cause-of-Death Disparities in the African Diaspora: Exploring Differences among Shared-Heritage Populations.

[18] Lori D. et al. Best Practices for Repositories: Collection, Storage, Retrieval and distribution of Biological Materials for research, ISBER third ed.

[19] Odedina FT et al., Prostate cancer health and cultural beliefs of black men: The Florida Prostate Cancer Disparity Project; 2011 Inf.Agent Cancer.

[20] Brureau L, et al.; Polymorphisms of estrogen metabolism-related genes and prostate cancer risk in two populations of African Ancestry. PLoS ONE 11(4): e0153609

[21] Akingbola TS et al. Comparison of patients from Nigeria and the USA highlights modifiable risk factors for sickle cell anemia complications;2014,Hemoglobin;236-243. 